\title{
Passung zum Beruf und die Wahl einer Aus- oder Weiterbildung
}

Christof Nägele, Simone Frey und Markus P. Neuenschwander

\section{Zusammenfassung}

Eine hohe Passung zum Beruf wird als ein wesentlicher Faktor für eine hohe Zufriedenheit und Verbundenheit mit dem Beruf angesehen. Eine sich verändernde Passung sollte deshalb auch Laufbahnentscheidungen erklären. Dabei kann die Planung einer Aus- oder Weiterbildung dazu genutzt werden, die (veränderte) Passung zum Beruf zu erhöhen. Deshalb kann angenommen werden, dass Personen mit einer geringen Passung zum Beruf eher eine Ausoder Weiterbildung planen. Die Passung einer Person zu ihrem Beruf und zur Weiterbildung kann einerseits als wahrgenommene Passung sowie als auf dem Person-Umwelt-Modell von Holland basierende Kongruenz dargestellt werden.

Es kann gezeigt werden, dass die wahrgenommene Passung sowohl die Zufriedenheit und Verbundenheit mit dem Beruf, wie auch die Planung einer Aus- oder Weiterbildung vorhersagt. Die Kongruenz Person-Beruf vermag dies nicht. Personen, die eine Aus- oder Weiterbildung planen, tun dies in der Regel so, dass diese besser zu ihnen passt als der aktuelle Beruf. Dies gilt jedoch nur für Personen mit einer tiefen Passung zu ihrem Beruf. Die Ergebnisse legen eine wichtige Rolle der wahrgenommenen Passung in der Bewertung und Steuerung der beruflichen Laufbahn nahe, stellen aber die Nützlichkeit einer auf Interessen beruhenden Bestimmung der Kongruenz in Frage.

\section{Schlagworte}

Laufbahn, Berufsbildung, Weiterbildung, Zufriedenheit, Verbundenheit 


\section{$1 \quad$ Passung zum Beruf und die Wahl einer Aus- oder Weiterbildung}

Eine kontinuierliche Aus- und Weiterbildung ist wichtig, und die Frage stellt sich bereits zu Beginn der beruflichen Laufbahn nach Abschluss einer beruflichen Grundbildung. Die Entscheidung, eine Aus- oder Weiterbildung zu planen oder zu besuchen, wird durch motivationale Faktoren (Neuenschwander, Nägele, \& Frey, n.d.) oder die Wahrnehmung von Chancen und Hindernissen, zum Beispiel in Zusammenhang mit der Vereinbarkeit von Familie und Arbeit (Frey, Neuenschwander \& Nägele, 2016) beeinflusst. In diesem Kapitel wird den Fragen nachgegangen, welchen Einfluss eine hohe Passung zum Beruf auf die Zufriedenheit und Verbundenheit mit der Arbeit hat und wie die Passung zum aktuellen Beruf die Planung einer Aus- oder Weiterbildung beeinflusst.

Eine hohe subjektive Passung zum Beruf ist nicht nur ein wichtiger Prädiktor für einen positiven Einstieg in eine neue Organisation (Nägele \& Neuenschwander, 2015), sondern auch eine Voraussetzung für die Entwicklung von Zufriedenheit und Verbundenheit mit dem Beruf (Holland, 1997; Super, 1980). Verändert sich die Passung zum Beruf, kann dies Veränderungen und Transitionen auslösen (Kristof-Brown, Zimmerman, \& Johnson, 2005). Wird die Passung geringer, besteht eine Möglichkeit darin, diese durch proaktives Verhalten (Grant \& Ashford, 2008) oder Job-crafting (Tims, Bakker, \& Derks, 2012) wieder zu verbessern. Wenn die eigenen Bedürfnisse und Interessen im aktuellen Beruf jedoch nicht mehr erfüllt werden können und somit kein Need-satisfaction -fit erzielt wird (Kristof-Brown et al., 2005), kann eine weitere Aus- oder Weiterbildung eine Option sein, um sich für einen anderen, passenderen Beruf zu qualifizieren. Durch die Wahl einer weiteren Aus- oder Weiterbildung passt eine Person ihre Umwelt so an, dass sie ihr optimale Entwicklungsmöglichkeiten bietet, im Sinne der Stage-environement-fit-Theorien (Eccles et al., 1993). Als aktive Gestalter ihrer eigenen Entwicklung steuern Personen so ihre berufliche Entwicklung (Savickas, 2012).

Zusammenfassend lässt sich festhalten, dass die Passung einer Person mit ihrem Beruf wichtig für die Beurteilung der aktuellen beruflichen Situation und auch für die Planung oder den Besuch einer Aus- oder Weiterbildung ist. Eine geringe Passung kann sich negativ auf die Befindlichkeit auswirken und Bildungsaktivitäten auslösen. Dies mit dem Ziel, die Passung (wieder) zu erhöhen. 


\subsection{Wahrgenommene Passung und Kongruenz Person-Beruf}

Passung ist als Übereinstimmung von Eigenschaften der Person mit Eigenschaften ihrer Umwelt definiert (Kristof, 1996). Die Passung einer Person zu ihrem Beruf kann anhand subjektiver oder objektiver Massen dargestellt werden (Judge, 2007).

Die wahrgenommene Passung entsteht aufgrund eines Vergleichs beruflicher und individueller Werte (Cable \& Judge, 1996). Eine hohe wahrgenommene Passung von Person und Beruf führt zu einer höheren beruflichen Zufriedenheit und Einschätzung des beruflichen Erfolgs. Bei einer guten Passung entwickelt sich die berufliche Laufbahn positiver (Hansen \& Lee, 2007; Neuenschwander, Gerber, Frank, \& Rottermann, 2012). Personen mit einer hohen Passung verbleiben eher in einer Organisation, sind leichter zu motivieren und erbringen bessere Leistungen (Kristof, 1996; Taris, Feij, \& Capel, 2006). Eine hohe Passung ist insgesamt ein guter Indikator für eine erfolgreich verlaufene Anpassung an den Beruf und die Organisation (Bauer \& Erdogan, 2011). Personen mit einer tiefen Passung haben hingegen ein erhöhtes Fluktuationsrisiko, sowohl in der beruflichen Grundbildung als auch in der Erwerbsarbeit (Stalder \& Schmid, 2016). Die wahrgenommene Passung verändert sich aufgrund neuer Erfahrungen (Nägele \& Neuenschwander, 2015).

Ein teilobjektives Mass der Passung ist die Kongruenz. Nach dem Person-Umwelt-Modell von Holland (1997) und Parsons (1909) können sowohl Personen als auch Berufe Typen zugeordnet werden. Der Personentyp beruht auf der Darstellung der Interessen einer Person, unterschiedliche berufliche Tätigkeiten ausführen zu wollen. Interessen werden als Ausdruck individueller Vorlieben verstanden. Die Typisierung der Berufe erfolgt anhand objektiver Kriterien durch Expertinnen und Experten (Jörin Fux, Stoll, Bergmann, Eder, \& Hell, 2012), in welchem Ausmass diese Tätigkeiten in dem entsprechenden Beruf vorkommen. Die Kongruenz ist der Grad der Übereinstimmung des Personen- und Umwelttyps (Jörin Fux et al., 2012). Zur Bestimmung der Kongruenz wurde der C-Index nach Brown (1994) berechnet, der die Anordnung der drei am stärksten ausgeprägten Interessendimensionen gemäss dem Hexagonmodell von Holland (1997) berücksichtigt (Jörin Fux, 2005).

\subsection{Zufriedenheit und Verbundenheit}

Zwei wichtige Ergebnisse einer erfolgreichen Anpassung an den Beruf sind die Zufriedenheit und die Verbundenheit mit dem Beruf (Bauer, Bodner, Erdogan, Truxillo, \& Tucker, 2007; Kammeyer-Mueller \& Wanberg, 2003). Zahlreiche Studien belegen, dass eine hohe Zufriedenheit als Ergebnis eines erfolgreichen Anpassungs- 
prozesses und als Voraussetzung für eine positive weitere Entwicklung wichtig ist (Keller \& Semmer, 2013).

Die Verbundenheit mit dem Beruf (Commitment) ist eine affektive Reaktion auf die aktuelle Situation (Cohen, 2007), die eng mit der Entwicklung einer beruflichen Identität verbunden ist (Stalder \& Nägele, 2015). Personen mit einer hohen Verbundenheit sind weniger abwesend, engagieren sich mehr in der Arbeit (Freund, 2005), leisten mehr und tragen sich weniger mit dem Gedanken, die Arbeit (K. Lee, Carswell, \& Allen, 2000) oder den Beruf (Hackett \& Lapierre, 2001) wechseln zu wollen.

\subsection{Planung einer weiteren Aus- oder Weiterbildung}

In dieser Studie ist die Planung einer formalen oder branchenspezifischen, umfangreichen Aus- oder Weiterbildung von Interesse. Der Fokus liegt auf der Frage, ob durch Bildungsentscheidungen Korrekturen im Bildungsverlauf vorgenommen werden, im Sinne der Selektions- oder Gravitationsthese (Heinz, 2000).

\subsection{Hypothesen}

Personen, die einen Beruf gewählt haben oder ausüben, der besser passt, sind insgesamt zufriedener und auch stärker mit ihrem Beruf verbunden.

Hypothese 1 Je höher die Passung einer Person mit ihrem Beruf ist, desto höher ist die Zufriedenheit mit dem Beruf. Dies trifft für a) die wahrgenommene Passung und für b) die Kongruenz zu.

Hypothese 2 Je höher die Passung einer Person mit ihrem Beruf ist, desto höher ist die Verbundenheit. Dies trifft für a) die wahrgenommene Passung und auch für b) die Kongruenz zu.

Eine weitere Aus- oder Weiterbildung schafft die Voraussetzung, eine nicht passende Situation zu verbessern (Neuenschwander \& Düggeli, 2013). Entsprechend sind die folgenden Hypothesen formuliert.

Hypothese 3 Eine tiefe a) wahrgenommene Passung oder b) Kongruenz führt dazu, dass die Personen eher eine weitere Aus- oder Weiterbildung planen. 
Hypothese 4 Personen planen eine weitere Ausbildung so, dass diese besser als ihr aktueller Beruf zu ihren eigenen Interessen passt. Dies trifft für a) die wahrgenommene Passung und b) die Kongruenz zu.

\section{$2 \quad$ Methode}

Die Daten für diese Analysen stammen aus der Studie „Berufliche Entscheidungen und Entwicklungsverläufe im Jugendalter und jungen Erwachsenenalter“ (BEN). BEN ist eine auf die Zeitspanne von 2012 bis 2017 angelegte Längsschnittstudie. Die Teilnehmenden wurden mittels Online-Fragebogen befragt (Neuenschwander \& Düggeli, 2013).

Für die vorliegenden Analysen wurden Personen ausgewählt, die 2012 kurz vor Abschluss der beruflichen Grundbildung waren (Kohorte 2) und Personen, die 2012 bereits seit einigen Jahren im Erwerbsleben standen (Kohorte 3). Eine vollständige Beschreibung der Studie und aller befragten Kohorten findet sich im zweiten Zwischenbericht zum Projekt BEN (Neuenschwander, Düggeli, Nägele, \& Frey, 2015).

In Welle I liegen Antworten von 775 Personen aus Kohorte 2 und von 342 Personen aus Kohorte 3 vor. In Welle II wurden die Teilnehmenden aus Welle I wieder zur Befragung eingeladen. Zusätzlich wurden für beide Kohorten zusätzliche Teilnehmende rekrutiert, um die Stichprobe zu vergrössern. In Welle II liegen von 1'318 Personen aus Kohorte 2 und von 1'413 Personen aus Kohorte 3 Antworten vor.

Die Längsschnittstichprobe besteht aus 485 Personen. In Kohorte 2 stehen Daten von 355 Personen und in Kohorte 3 von 130 Personen zur Verfügung.

Die Personen in der Kohorte 2 waren im Jahr 2014 im Durchschnitt 22.4 Jahre alt $(S D=3.4$ Jahre), in der Kohorte 3 im Durchschnitt 28.1 Jahre alt $(S D=2.2$ Jahre).

\subsection{Messinstrumente}

Die wahrgenommene Passung wurde mit drei Items gemessen (Neuenschwander \& Frank, 2009), z. B. „Meine Arbeit passt zu meiner Person“, auf einer Antwortskale von 1 „stimmt überhaupt nicht“ bis 6 "stimmt voll und ganz". In Welle I wurde für Kohorte 2 anstelle von „Meine Arbeit..." nach „Meine Lehre..." gefragt. Die interne Konsistenz der Skala war in Welle 1 für die Passung mit der Lehre Cronbach's Alpha $=.90$, für die Passung mit dem Beruf Cronbach's Alpha $=.88$, in Welle II Cronbach's Alpha $=.88, N=1$ '781. In Welle II wurde zusätzlich die wahrgenommene Passung 
zur Aus- oder Weiterbildung erfasst. Die interne Konsistenz der Skala war in Welle II Cronbach's Alpha $=.81$.

Die Kongruenz zwischen Person und Beruf wurde mittels des C-Index nach Brown und Gore (1994) berechnet, der auf einem gewichteten Vergleich der beiden Primär-, Sekundär- und Tertiärtypen im Verhältnis zu deren Lage auf dem Hexagon beruht (Jörin Fux, 2005). Die Personentypen wurden mittels des Allgemeinen Interesse-Struktur-Tests (AIST-R) erhoben (Bergmann, 2004). Die Umwelttypen (Berufsprofile) wurden anhand der Expertenratings in Explorix (Jörin Fux et al., 2012) kodiert.

Als Outcomes werden in der vorliegenden Studie die berufliche Zufriedenheit und die Verbundenheit mit dem Beruf untersucht.

In Welle I wurde nach der Zufriedenheit mit der Lehre und der Berufsfachschule (Kohorte 2), resp. dem Betrieb und dem Beruf (Kohorte 3) gefragt, in Anlehnung an Neuenschwander et al. (2013a). Die berufliche Zufriedenheit ist der Durchschnittswert der je bereichspezifischen Zufriedenheiten. In Welle II wurde die Zufriedenheit für Kohorte 2 und Kohorte 3 für den Beruf und den Betrieb erfasst und zu einem Mass der beruflichen Zufriedenheit zusammengefasst. Die berufliche Zufriedenheit wurde in Welle I auf einer 6-stufigen Antwortskale von 1 „sehr unzufrieden“" bis 6 ,sehr zufrieden“ "erfasst. In Welle II wurde eine 7-stufige Antwortskale verwendet. Diese 7-stufige Antwortskala wurde für die Analysen in eine 6-stufige Antwortskala transformiert.

Die Verbundenheit mit dem Beruf (Commitment) wurde in Welle I mit drei Items gemessen, z.B. „Diese Ausbildung, resp. Arbeit zu haben ist wichtig“, auf einer Antwortskale von 1 „stimmt überhaupt nicht“ bis 6 „stimmt voll und ganz" (Neuenschwander, Gerber, Frank, Singer, \& Bosshard, 2013a). Die interne Konsistenz der Skala war Cronbach's Alpha $=.79$. In Welle II wurde die Verbundenheit mit dem Beruf (Commitment) in Anlehnung an Allen und Meyer (1990), in einer Adaptation von Nübling (2005) mit drei Items gemessen, z. B. „Stolz, diesem Betrieb anzugehören“, auf einer Antwortskale von 1 ,stimmt überhaupt nicht“ bis 6 ,stimmt voll und ganz". Die interne Konsistenz der Skala war Cronbach's Alpha $=.85$.

Die Planung einer Aus- oder Weiterbildung wurde in Welle I für die Kohorte 2 mit zwei Fragen erfasst (Antwortoptionen ja-nein): „Erwägen Sie eine Aus- oder Weiterbildung?" und „Haben Sie diese Aus- oder Weiterbildung verbindlich geplant?" (Neuenschwander, Hermann, Frank, \& Faschinger, 2013b). Die Planung einer weiteren Ausbildung wurde für Kohorte 3 in einer Frage mit den Antwortoptionen erfasst: „Im Moment habe ich keine konkreten Pläne, eine Aus- oder Weiterbildung zu beginnen“, „Ich plane eine Aus- oder Weiterbildung zu beginnen“ und „Ich werde sicher eine Aus- oder Weiterbildung beginnen "(Neuenschwander, Hermann, Frank, \& Faschinger, 2013b). Diese Variable wurde in zwei Kategorien zusammengefasst, 
1 „keine Planung“ und 2 „Planung oder beginnen“. In Welle II wurde die geplante weitere Ausbildung für beide Kohorten gleich erfragt wie bei Kohorte 3 in Welle I.

Bildungsentscheidungen werden durch das Geschlecht (Berweger, Kappler, Keck Frei, \& Bieri Buschor, 2015) und strukturelle Merkmale beeinflusst. Das über die Kohorte erfasste strukturelle Merkmal ist der aktuelle Stand der Laufbahn. Dies ist für Kohorte 2 der Übergang von der beruflichen Grundbildung in die berufliche Tätigkeit und für Kohorte 3 die allfällige Planung einer weiteren Aus- oder Weiterbildung.

\section{$3 \quad$ Resultate}

\subsection{Deskriptive Ergebnisse}

In Tabelle 1 sind die Mittelwerte, Standardabweichungen und Korrelationen für die Welle I, die Welle II und den Längsschnitt dokumentiert.

Die Mittelwerte der Personen, die sich an beiden Wellen beteiligten, unterscheiden sich von denen, die sich nur an Welle I, respektive nur an Welle II beteiligten, teilweise systematisch. Die Effektstärken sind jedoch gering (Cohen's $d<.30$ ). Die Personen der Längsschnittstichprobe waren mehr mit ihrem Beruf verbunden, hatten eine etwas höhere Kongruenz mit der Aus- oder Weiterbildung und waren auch etwas zufriedener mit der Arbeit als die übrigen Teilnehmenden in Welle I oder Welle II.

Tab. 1 Deskriptive Ergebnisse und Korrelationen

\begin{tabular}{|c|c|c|c|c|c|c|c|c|c|c|c|}
\hline Welle I/Welle II & MW & SD & 1 & 2 & 3 & 4 & 5 & 6 & 7 & 8 & 9 \\
\hline 1 Geschlecht & $1.49 / 1.42$ & $.50 / .49$ & 1 & -.01 & -.06 & $-.26^{* *}$ & $-.08^{*}$ & · & $-.12^{* *}$ & & . \\
\hline 2 Kohorte & $2.31 / 2.52$ & $.46 / .50$ & .02 & 1 & $.09^{* *}$ & -.20 & $.60^{* *}$ & . & $-.10^{* *}$ & & \\
\hline 3 Zufriedenheit & $4.76 / 5.10$ & $.75 / 1.18$ & .04 & -.02 & 1 & $.19^{* *}$ & $.09^{* *}$ & . & $.60^{* *}$ & & . \\
\hline 4 Verbundenheit & $5.04 / 4.37$ & $.89 / 1.04$ & .05 & -.01 & $.44^{* *}$ & 1 & $.73^{\star}$ & . & $.22^{* *}$ & & . \\
\hline 5 Planung & $1.41 / 1.46$ & $.49 / .50$ & $.05^{*}$ & $-.21^{* *}$ & -.04 & -.02 & 1 & . & -.03 & & . \\
\hline 6 Kongruenz &.$/ 10.91$ &.$/ 3.66$ & .02 & .03 & .04 & $.09^{* *}$ & -.02 & 1 & & & . \\
\hline $\begin{array}{l}7 \text { Wahrgenom- } \\
\text { mene Passung }\end{array}$ & $4.85 / 4.62$ & $.93 / 1.01$ & .02 & .03 & $.46^{* *}$ & $.67^{* *}$ & $-.08^{* *}$ & $.11^{* *}$ & 1 & & . \\
\hline 8 Differenz W & .1 .52 &.$/ 1.12$ & -.07 & .03 & $-.35^{* *}$ & $-.56^{* *}$ & .08 & $-.13^{*}$ & $-.78^{* *}$ & 1 & . \\
\hline 9 Differenz K & .1 .53 &.$/ 4.41$ & -.01 & -.07 & -.05 & -.04 & -.08 & $-.59^{* *}$ & -.08 & .01 & 1 \\
\hline
\end{tabular}

Bemerkung. Welle I oberhalb der Diagonale, N zwischen 985 und 1055; Welle II (kursiv) unterhalb der Diagonale $N$ zwischen 368 und 2635. ${ }^{\star} p<.05,{ }^{* *} p<.01$, ,." $=$ keine Daten verfügbar 


\begin{tabular}{lccccccccc}
\hline Längsschnitt & \multicolumn{1}{c}{ Welle II } \\
\hline Welle I & MW & \multicolumn{1}{c}{ SD } & 1 & 2 & 3 & \multicolumn{1}{c}{4} & \multicolumn{1}{c}{5} & \multicolumn{1}{c}{6} & \multicolumn{1}{c}{7} \\
\hline 1 Geschlecht & 1.45 & .50 & 1 & .09 & .03 & $.13^{*}$ & -.02 & .01 & $-.13^{* *}$ \\
2 Kohorte & 2.27 & .44 & .09 & 1 & -.03 & .05 & -.01 & .01 & .08 \\
3 Zufriedenheit & $4.76 / 5.28$ & $.70 / 1.06$ & -.02 & -.05 & $.21^{* *}$ & $.20^{* *}$ & -.05 & .01 & $.27^{* *}$ \\
4Verbundenheit & $5.11 / 4.27$ & $.81 / 1.02$ & $-.25^{* *}$ & -.04 & .04 & -.10 & .03 & -.13 & -.05 \\
5 Planung & $1.44 / 1.48$ & $.50 / .50$ & -.02 & $-.63^{* *}$ & -.02 & .05 & -.04 & .02 & .08 \\
6 Kongruenz &.$/ 11.42$ &.$/ 3.50$ &. &. &. &. &. &. &. \\
7 Wahrgenom- & $4.90 / 4.59$ & $.91 / .92$ & $-.10^{*}$ & $-.13^{* *}$ & .10 & .08 & -.04 & .17 & .01 \\
mene Passung & & & & & & & & & \\
\hline
\end{tabular}

Bemerkung. Kursiv = Welle II, N zwischen 995 und 116; Längsschnitt N zwischen 159 und 485. ${ }^{\star} \mathrm{p}<.05,{ }^{* *} \mathrm{p}<.01, .=$ keine Daten verfügbar

\subsection{Planung einer Aus- oder Weiterbildung}

In Tabelle 2 ist aufgeführt, wie viele Personen eine Aus- oder Weiterbildung in Welle I und Welle II planten. Auffällig ist, dass in Welle I in der Kohorte 2 deutlich mehr Personen keine Aus- oder Weiterbildung planten als erwartet $(N=611$, erwartet 459, adj. Residual -20.0). In Welle II planten hingegen mehr Personen der Kohorte 2 als erwartet eine Aus- oder Weiterbildung $(N=665$, erwartet 535, adj. Residual 5.6).

Tab. 2 Planung einer weiteren Aus- oder Weiterbildung

\begin{tabular}{llrrrr}
\hline & Planung & \multicolumn{2}{c}{ Welle I } & \multicolumn{2}{c}{ Welle II } \\
\hline Kohorte 2 & Nein & 611 & $79 \%$ & 512 & $44 \%$ \\
& Ja & 164 & $21 \%$ & 665 & $56 \%$ \\
Total & & 775 & $100 \%$ & $1^{\prime} 117$ & $100 \%$ \\
\hline Kohorte 3 & Nein & 51 & $15 \%$ & 817 & $65 \%$ \\
& Ja & 291 & $85 \%$ & 446 & $35 \%$ \\
\hline Total & & 342 & $100 \%$ & 1 '263 & $100 \%$ \\
\hline
\end{tabular}

Bemerkungen. Welle I: Chi-Quadrat $(1)=401.67, p<.01$. Welle II: Chi-Quadrat $(1)=110.27, p<.01$

In Welle I planten insgesamt mehr Frauen als erwartet eine weitere Aus- oder Weiterbildung $(N=236$, erwartet 217 , adj. Residual 2.4), was sich aber in Welle II umkehrte. Es gab in Welle II mehr Männer als erwartet, die eine weitere Aus- oder Weiterbildung planten ( $N=462$, erwartet 435, adj. Residual 2.3) (Tabelle 3). 
Tab. 3 Planung nach Geschlecht

\begin{tabular}{lllrrr}
\hline & Planung & Welle I & \multicolumn{3}{c}{ Welle II } \\
\hline \multirow{2}{*}{ Frauen } & nein & 294 & $56 \%$ & 792 & $56 \%$ \\
& ja & 236 & $44 \%$ & 613 & $44 \%$ \\
\hline \multirow{2}{*}{ Total } & & 530 & $100 \%$ & 1 '405 & $100 \%$ \\
\hline \multirow{2}{*}{ Männer } & nein & 322 & $63 \%$ & 493 & $52 \%$ \\
& ja & 190 & $37 \%$ & 462 & $48 \%$ \\
\hline Total & & 512 & $100 \%$ & 955 & $100 \%$ \\
\hline
\end{tabular}

Bemerkungen. Welle I: Chi-Quadrat $(1)=5.93, p<.02$. Welle II: Chi-Quadrat $(1)=110.27, p<.01$

\subsection{Prüfung der Hypothesen}

In Hypothese 1 ist formuliert, dass eine höhere Passung einer Person mit ihrem Beruf positiv mit der beruflichen Zufriedenheit zusammenhängt. Die Ergebnisse in Tabelle 4 zeigen, dass die berufliche Zufriedenheit in Welle I mit der wahrgenommenen Passung und der Kohorte zusammenhing. In Welle II zeigte sich ein Effekt der wahrgenommenen Passung, und im Längsschnitt war es die wahrgenommene Passung in

Tab. 4 Prädiktoren der beruflichen Zufriedenheit

\begin{tabular}{|c|c|c|c|c|c|c|c|c|c|c|c|c|c|c|c|}
\hline & \multicolumn{5}{|c|}{ Welle I } & \multicolumn{5}{|c|}{ Welle II } & \multicolumn{5}{|c|}{ Längsschnitt } \\
\hline & B & S.F. & Beta & $\mathrm{t}$ & sig. & B & S.F. & Beta & $\mathrm{t}$ & sig. & B & S.F. & Beta & $\mathrm{t}$ & sig. \\
\hline Geschlecht & .03 & .04 & .02 & .80 & .42 & .07 & .06 & .03 & 1.05 & .29 & .12 & .19 & .05 & .64 & .52 \\
\hline Kohorte & .25 & .04 & .15 & 5.93 & $<.01$ & -.01 & .06 & -.01 & -.22 & .83 & -.04 & .20 & -.02 & -.21 & .84 \\
\hline $\begin{array}{l}\text { Berufliche } \\
\text { Zufrieden- } \\
\text { heit WI }\end{array}$ & & & & & & & & & & & .36 & .18 & .22 & 1.98 & .05 \\
\hline $\begin{array}{l}\text { Wahrge- } \\
\text { nommene } \\
\text { Passung } \\
\text { WI }\end{array}$ & .50 & .02 & .62 & 24.61 & $<.01$ & & & & & & -.18 & .15 & -.14 & -1.23 & .22 \\
\hline $\begin{array}{l}\text { Kongruenz } \\
\text { WII }\end{array}$ & & & & & & -.01 & .01 & -.02 & -.63 & .53 & -.03 & .03 & -.10 & -1.29 & .20 \\
\hline $\begin{array}{l}\text { Wahrge- } \\
\text { nommene } \\
\text { Passung } \\
\text { WII }\end{array}$ & & & & & & .53 & .03 & .45 & 17.31 & $<.01$ & .41 & .11 & .33 & 3.87 & $<.01$ \\
\hline
\end{tabular}

Bemerkungen.; Welle I $N=983: F(3,979)=207.22, p<.01, \mathrm{R}^{2}=.39$. Für Welle I liegt kein Mass für die Kongruenz vor, die Interessen wurden ab Welle II erfasst. Welle II $N=1^{\prime} 213$ : $F(4,1208)=75.94, p<.01, \mathrm{R}^{2}=.20$. Längsschnitt $N=144: F(6,137)=4.72,<=.01, \mathrm{R}^{2}=.14$. S.F.: Standardfehler 
Welle II, welche zur Erklärung der Varianz in der beruflichen Zufriedenheit beitrug. Die Teilnehmenden der Kohorte 2 waren zum Zeitpunkt der Befragung kurz vor Abschluss ihrer Lehre. Dies kann erklären, weshalb die Kohorte einen Einfluss auf die berufliche Zufriedenheit hatte. Im Längsschnitt war ein wichtiger Prädiktor der beruflichen Zufriedenheit in Welle II die berufliche Zufriedenheit in Welle I.

Die Hypothese 1a kann nur teilweise bestätigt werden. Querschnittlich erklärt die wahrgenommene Passung die Zufriedenheit mit dem Beruf, längsschnittlich jedoch nicht. Die Kongruenz Person-Beruf hat keinen Einfluss auf die Zufriedenheit, Hypothese $1 \mathrm{~b}$ ist nicht bestätigt.

In Hypothese 2 ist formuliert, dass eine höhere Passung einer Person mit ihrem Beruf mit einer höheren Bindung an den Beruf (Commitment) zusammenhängt. Die Ergebnisse in Tabelle 5 zeigen, dass die Verbundenheit mit dem Beruf in Welle I mit der wahrgenommenen Passung und dem Geschlecht zusammenhing. Das negative Vorzeichen beim Beta des Geschlechts bedeutet, dass die Männer in Welle I eher mit ihrem Beruf verbunden waren als die Frauen. In Welle II hing die Verbundenheit mit dem Beruf mit der wahrgenommenen Passung zusammen, wie auch im Längsschnitt. Im Längsschnitt ist auffallend, dass keine Prädiktoren aus der Welle I zur Vorhersage der Verbundenheit mit dem Beruf signifikant wurde.

Tab. 5 Prädiktoren der Verbundenheit mit dem Beruf

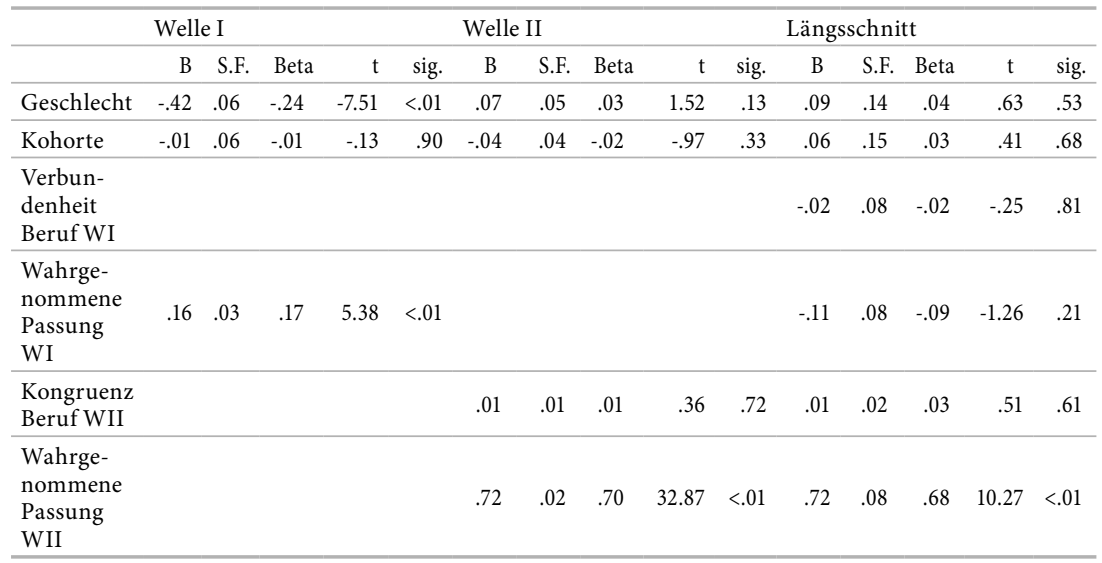

Bemerkung. Welle I $N=924: F(2,921)=33.46, p<.01, \mathrm{R}^{2}=.07$; Modell 2: $F(3,920)=32.62$, $p<.01, \mathrm{R}^{2}=.09$, Welle II $N=1$ '214: $F(4,1209)=275.51, p<.01, \mathrm{R}^{2}=.48$. Längsschnitt $N=142: F(6,135)=20.07,<=.01, \mathrm{R}^{2}=.45$. S.F.: Standardfehler 
Die Hypothese 2a kann nur teilweise bestätigt werden. Querschnittlich erklärt die wahrgenommene Passung die Verbundenheit mit dem Beruf, längsschnittlich jedoch nicht. Die Kongruenz Person-Beruf hat keinen Einfluss auf die Verbundenheit, Hypothese $2 \mathrm{~b}$ ist nicht bestätigt.

In Hypothese 3 ist formuliert, dass eine tiefe wahrgenommene Passung oder Kongruenz dazu führen, dass die Personen eher eine Aus- oder Weiterbildung planen. Die Ergebnisse in Tabelle 6 zeigen, dass in Welle I die Planung einer weiteren Aus- und Weiterbildung vor allem durch das Geschlecht bestimmt war. In Welle I planten Frauen mit einer höheren Wahrscheinlichkeit eine Aus- oder Weiterbildung als die Männer. In Welle II dreht sich dieser Geschlechtseffekt um. Nun waren es die Männer, die eher eine Aus- oder Weiterbildung planten. Zudem führte eine höhere wahrgenommene Passung dazu, eher keine Aus- oder Weiterbildung zu planen. Längsschnittlich kann kein Prädiktor einen Beitrag zur Erklärung der Planung leisten.

Tab. 6 Prädiktoren der Planung einer weiteren Aus- oder Weiterbildung

\begin{tabular}{|c|c|c|c|c|c|c|c|c|c|c|c|c|}
\hline & \multicolumn{4}{|c|}{ Welle I } & \multicolumn{4}{|c|}{ Welle II } & \multicolumn{4}{|c|}{ Längsschnitt } \\
\hline & $\operatorname{Exp}(B)$ & $\begin{array}{r}\text { CFI } \\
95 \%\end{array}$ & & sig. & $\operatorname{Exp}(B)$ & $\begin{array}{r}\text { CFI } \\
95 \%\end{array}$ & & sig. & $\operatorname{Exp}(B)$ & $\begin{array}{r}\text { CFI } \\
95 \%\end{array}$ & & sig. \\
\hline Geschlecht & 1.78 & 1.25 & 2.53 & $<.01$ & .77 & .60 & .98 & .03 & .52 & .25 & 1.06 & .07 \\
\hline Kohorte & .01 & .00 & .02 & $<.01$ & 2.44 & 1.92 & 3.08 & $<.01$ & 1.28 & .42 & 3.92 & .66 \\
\hline Planung WI & & & & & & & & & .76 & .27 & 2.13 & .60 \\
\hline Passung WI & 1.12 & .91 & 1.38 & .27 & & & & & .63 & .41 & .96 & .03 \\
\hline $\begin{array}{l}\text { Kongruenz } \\
\text { Beruf WII }\end{array}$ & & & & & .99 & .96 & 1.03 & .72 & 1.05 & .95 & 1.15 & .37 \\
\hline $\begin{array}{l}\text { Passung } \\
\text { WII }\end{array}$ & & & & & .88 & .78 & 1.03 & .04 & .98 & .98 & 1.43 & .89 \\
\hline
\end{tabular}

Bemerkung. Welle I $N=985:-2$ Log likelihood $=826.23$, Chi-Quadrat $(3)=517.85, p<.01$, Nagelkerke $\mathrm{R}^{2}=.55$. Welle II $N=1213:-2$ Log likelihood = 1605.93, Chi-Quadrat $(4)=64.73$, $p<.01$, Nagelkerke $\mathrm{R}^{2}=.07$. Längsschnitt II $N=1213:-2$ Log likelihood $=191.10$, Chi-Quadrat $(6)=8.27, p=.22$, Nagelkerke $\mathrm{R}^{2}=.07$

Hypothese 3a wurde teilweise bestätigt, da eine tiefere wahrgenommene Passung in Welle II die Wahrscheinlichkeit erhöhte, eine Aus- oder Weiterbildung zu planen. Hypothese $3 \mathrm{~b}$ kann nicht bestätigt werden, da die Kongruenz Person-Beruf keinen Einfluss auf die Planung einer Aus- oder Weiterbildung hatte.

In Hypothese 4 wird formuliert, dass Personen eine Aus- oder Weiterbildung so wählen, dass diese passender als der aktuelle Beruf ist. Dazu wurde ein Differenzwert der Passung zwischen der Aus- und Weiterbildung und der Passung mit 
dem Beruf berechnet. Je grösser diese Differenz ist, desto unähnlicher sind sich der Beruf und die Aus- oder Weiterbildung. Der Differenzwert wurde zuerst für die wahrgenommene Passung der Aus- oder Weiterbildung und der wahrgenommenen Passung zum Beruf bezüglich der geplanten Aus- und Weiterbildung berechnet. Der Differenzwert lag bei $M W=.52, S D=1.12, N=483$. Zweitens wurde zur Berechnung des Differenzwerts für die Kongruenz Person-Beruf die Kongruenz Person-besuchte Aus- oder Weiterbildung berechnet. Dieser Differenzwert liegt bei $M W=.53, S D=4.41, N=383$. Im Durchschnitt planten die befragten Personen also eher eine Aus- oder Weiterbildung mit einer höheren Kongruenz.

In Tabelle 7 sind zuerst die Ergebnisse zur Erklärung der Differenzwerte der wahrgenommenen Passung aufgeführt. Die Differenzwerte können durch das Geschlecht, die Kohorte und die Kongruenz Person-Beruf nicht vorhergesagt werden. Signifikant wird die wahrgenommene Passung. Personen mit einer tiefen Passung planen eine Aus- oder Weiterbildung, die besser zu ihnen passt. Dies gilt jedoch nur bis zu einem Grenzwert. Aufgrund der Regressionsgleichung planten Personen mit einer wahrgenommenen Passung von mehr als 5.21 eine Aus- oder Weiterbildung, die weniger gut passte.

In Tabelle 7 sind zweitens die Ergebnisse zur Erklärung der Differenz der Kongruenz der besuchten Aus- oder Weiterbildung und des aktuellen Berufs aufgeführt. Diese Differenz wird durch die Kongruenz Person-Beruf erklärt. Sie wird kleiner, je grösser die Kongruenz Person-Beruf ist. Personen mit einer tiefen Kongruenz planten also eine Aus- oder Weiterbildung, die deutlich kongruenter war als ihr Beruf. Aufgrund der Regressionsgleichung kann gezeigt werden, dass Personen mit einer Kongruenz Person-Beruf von weniger als 11.5 eine weitere Aus- oder Weiterbildung planten, die besser passte, bei einer Kongruenz von mehr als 11.5 aber eine weitere Aus- oder Weiterbildung, die weniger gut passte.

Tab. 7 Prädiktoren des Differenzwerts

\begin{tabular}{lcccccccccc}
\hline Welle II & \multicolumn{1}{c}{ Differenzwert Wahrgenommene Passung } & \multicolumn{5}{c}{ Differenzwert Kongruenz } \\
\hline & B & S.F. & Beta & $\mathrm{t}$ & sig. & B & S.F. & Beta & $\mathrm{t}$ & sig. \\
\hline Geschlecht & -.04 & .08 & -.02 & -.46 & .64 & .06 & .40 & .01 & .15 & .47 \\
\hline Kohorte & .10 & .01 & .05 & 1.27 & .20 & -.29 & .39 & -.03 & -.89 & .39 \\
\hline $\begin{array}{l}\text { Kongruenz } \\
\text { Beruf WII }\end{array}$ & .01 & .01 & .05 & 1.28 & .20 & -.68 & -.05 & -.55 & -12.59 & $<.01$ \\
\hline $\begin{array}{l}\text { Passung } \\
\text { Beruf WII }\end{array}$ & -.83 & .04 & -.78 & -21.44 & $<.01$ & -.17 & .19 & -.04 & -.87 & .39 \\
\hline Konstante & 3.96 & .29 & & 13.09 & $<.01$ & 9.20 & 1.48 & & 6.22 & $<.01$ \\
\hline
\end{tabular}

Bemerkung. Wahrgenommene Passung: $N=336: F(4,331)=118.29, p<.01, \mathrm{R}^{2}=.58$. Kongruenz $N=336: F(4,361)=41.22, p<.01, \mathrm{R}^{2}=.31$ 
Die Hypothesen 4a und 4b können teilweise bestätigt werden, da Personen eine weitere Aus- oder Weiterbildung wählten, die besser passte oder kongruenter war. Dies gilt jedoch nur bis zu einem bestimmten Grenzwert. Wird dieser überschritten, wählten die Personen weniger passende oder weniger kongruente Aus- oder Weiterbildungen.

\section{$4 \quad$ Diskussion}

Die auf dem Person-Umwelt-Modell (Holland, 1997; Super, 1980) basierende Annahme, dass eine hohe Kongruenz zu einer höheren Zufriedenheit und einer stärkeren Verbundenheit führt, konnte nicht bestätigt werden. Hingegen konnte ein deutlicher Zusammenhang mit der subjektiven, wahrgenommenen Passung (Kristof-Brown et al., 2005) gezeigt werden. Im Längsschnitt zeigte sich, dass die Zufriedenheit mit dem Beruf in Welle II von der Zufriedenheit in der Welle I abhing, was auf eine Stabilität der Zufriedenheit mit dem Beruf über die Zeit hinweist (Keller \& Semmer, 2013). Die berufliche Zufriedenheit und Verbundenheit scheinen sich also nicht aufgrund dispositionaler Faktoren (Interessen), sondern aufgrund einer subjektiven Einschätzung der Qualität des Anpassungsprozesses zu entwickeln (Nägele \& Neuenschwander, 2016). Der fehlende Einfluss der Verbundenheit mit dem Beruf in Welle I auf die Verbundenheit mit dem Beruf in Welle II ist durch die Veränderung des Messinstruments gut zu erklären.

Weder die wahrgenommene Passung noch die Kongruenz sind gute Prädiktoren der Planung einer weiteren Aus- oder Weiterbildung. Die Planung einer Aus- und Weiterbildung unterscheidet sich in Welle I und Welle II. In Welle I scheinen vor allem die Männer der Kohorte 2 die Planung der Aus- oder Weiterbildung aufzuschieben. Dies kann damit erklärt werden, dass einige junge Männer nach Abschluss der beruflichen Grundbildung zuerst den Militärdienst absolvieren und die Planung einer weiteren Aus- oder Weiterbildung aufschieben. Frauen finden sich eher in Gesundheitsberufen, in denen eine frühe und kontinuierliche formale Ausbildung einen höheren Stellenwert hat als in vielen handwerklichen Berufen (Trede, 2016). Wenn ein Effekt auf die Planung einer Aus- oder Weiterbildung auftritt, dann ist es ein Effekt der wahrgenommenen Passung, nicht aber der Kongruenz Person-Beruf. Je höher die Passung, desto weniger wahrscheinlich ist die Planung. In Welle II ist dieser Effekt der aktuellen Passung auf die Planung einer Aus- oder Weiterbildung deutlich zu sehen. Im Längsschnitt lässt sich die Planung nicht vorhersagen. Das heisst, dass vor allem eine aktuell als schlechte wahrgenommen Passung Planungsaktivitäten auslöst. 
Personen mit einer insgesamt tiefen Passung zum Beruf oder mit einer geringen Kongruenz Person-Beruf wählen eine Aus- oder Weiterbildung so, dass diese besser passt, resp. kongruenter ist. Personen mit einer bereits hohen wahrgenommenen Passung oder hohen Kongruenz wählen eine Aus- oder Weiterbildung, die eher weniger zu ihnen passte. Dies heisst, dass nur Personen mit einem unter einem bestimmten Grenzwert liegenden Niveau der Passung versuchen, eine geringe Passung Person-Beruf zu verbessern. Dieses Ergebnis kann aufgrund der vorliegenden Daten unterschiedlich interpretiert werden. So ist es möglich, dass Personen mit einer bereits sehr hohen Passung zum Beruf kaum eine Aus- oder Weiterbildung finden können, die noch besser passt. Es ist auch möglich, dass diese Personen aufgrund biographischer Ereignisse gezwungen sind, ihren Beruf zu wechseln. Dies zum Beispiel aus gesundheitlichen oder familiären Gründen. Weitere Analysen mit Daten zum Bildungsverlauf, die aktuell noch nicht vorliegen, werden hier Antworten ermöglichen.

Die querschnittlichen und längsschnittlichen Befunde unterscheiden sich teilweise. Da die beiden Erhebungszeitpunkte zwei Jahre auseinanderliegen, kann dies als Hinweis interpretiert werden, dass die Planung einer Aus- oder Weiterbildung bei den Jugendlichen und jungen Erwachsenen stark auch von aktuellen Ereignissen beeinflusst wird und nicht von Ereignissen und Einschätzungen von vor zwei Jahren.

Einschränkungen. Es liegen nicht zu allen Variablen Daten im Längsschnitt vor, so dass einige Ergebnisse auf querschnittlichen Analysen beruhen. Die befragten Personen bilden eine relativ homogene Gruppe mit einer im Durchschnitt hohen Passung und Kongruenz zu ihrem Beruf. Die Längsschnittstichprobe ist im Vergleich zur Stichprobe in Welle I und Welle II relativ klein, was geschlechts-, und kohortenspezifische Auswertungen erschwert. Deshalb werden in diesem Kapitel keine Hypothesen zu Geschlecht und Kohorte formuliert.

Zusammenfassung. Es scheint wichtig, der subjektiven Einschätzung der Passung zum Beruf einen hohen Stellenwert zu geben. Personen mit einer tiefen wahrgenommenen Passung sind weniger zufrieden und weniger an den Beruf gebunden, und sie planen eher eine weitere Aus- und Weiterbildung. Wenn Personen eine Aus- oder Weiterbildung planen, dann planen sie diese so, dass sie besser zu ihnen passt, sofern die Passung Person-Beruf tief ist. Die besuchte Weiterbildung passt besser zu den eigenen Interessen, wenn sich diese Interessen und der bisherige Beruf schlecht entsprechen. Bei einer hohen wahrgenommenen Passung oder Kongruenz Person-Beruf findet sich dieser Effekt nicht mehr. Die Planung einer Aus- und Weiterbildung ist von der aktuellen Dynamik im Beruf abhängig. Wenn eine Aus- oder Weiterbildung geplant wird, so erfolgt dies aufgrund zeitlich naher Ereignisse und Einschätzungen. Das heisst aber auch, dass so lange die Situation als subjektiv passend wahrgenommen wird, die Planung einer Aus- oder Weiterbildung 
weniger wahrscheinlich ist. Erst wenn die Situation als unpassend wahrgenommen wird, kommt es zu einer Planung. Bei Personen, die eine Aus- oder Weiterbildung umsetzen, wird diese so gewählt, dass sie kongruenter ist, das heisst, besser zu den Interessen passt. Das heisst, dass die Planung einer Aus- oder Weiterbildung wahrscheinlich durch eine subjektiv wahrgenommene Verschlechterung der Passung ausgelöst wird. Welche Aus- oder Weiterbildung gemacht wird, wird dann aber durch die Interessen mit beeinflusst.

Die Passung wird als ein wichtiger Faktor diskutiert, der nicht nur die Berufsfindung, sondern auch die Entwicklung im Beruf erklären soll. Die in diesem Kapitel präsentierten Ergebnisse zeigen, dass die wahrgenommene Passung, also die Selbsteinschätzung, ob man sich im passenden Beruf oder der passenden Ausbildung befindet, einen sehr grossen Einfluss auf die Zufriedenheit und die Verbundenheit mit dem Beruf hat. Die Kongruenz spielt dabei keine Rolle.

\section{Literatur}

Allen, N. J., \& Meyer, J. P. (1990). The measurement and antecedents of affective, continuance and normative commitment to the organization. Journal of Occupational Psychology, 63, 1-18.

Bauer, T. N., \& Erdogan, B. (2011). Organizational socialization: The effective onboarding of new employees. In S. Zedeck (Ed.), APA handbook of industrial and organizational psychology (Vol. III, pp. 51-64). Washington, DC: American Psychological Association. http://doi.org/10.1037/12171-002

Bauer, T. N., Bodner, T., Erdogan, B., Truxillo, D. M., \& Tucker, J. S. (2007). Newcomer adjustment during organizational socialization: A meta-analytic review of antecedents, outcomes, and methods. Journal of Applied Psychology, 92(3), 707-721. http://doi. org/10.1037/0021-9010.92.3.707

Bergmann, C. (2004). Berufswahl. In H. Schuler (Hrsg.), Organisationspsychologie - Grundlagen und Personalpsychologie (S. 343-388). Göttingen, D: Hogrefe Verlag für Psychologie.

Berweger, S., Kappler, C., Keck Frei, A., \& Bieri Buschor, C. (2015). Geschlechtsuntypische Laufbahnpläne. Schweizerische Zeitschrift für Bildungswissenschaften, 37(2), 321-339.

Brown, S. D., \& Gore, P. A., Jr. (1994). An evaluation of interest congruence indices: Distribution characteristics and measurement properties. Journal of Vocational Behavior, 45, 310-327.

Cable, D. M., \& Judge, T. A. (1996). Person-organization fit, job choice decisions, and organizational entry. Organizational Behavior and Human Decision Processes, 67, 294-311. http://doi.org/10.1037/0021-9010.92.5.1446

Cohen, A. (2007). Dynamics between occupational and organizational commitment in the context of flexible labor markets: A review of the literature and suggestions for a future research agenda. Bremen, D: Universität Bremen, Institut Technik und Bildung. 
Eccles, J. S., Midgley, C., Wigfield, A., Buchanan, C. M., Reuman, D., Flanagan, C., \& Mac Iver, D. (1993). Development during adolescence: The impact of stage-environment fit on young adolescents' experiences in schools and in families. American Psychologist, $48(2), 90-101$.

Freund, A. (2005). Commitment and job satisfaction as predictors of turnover intentions among welfare workers. Administration in Social Work, 29(2), 5-21. http://doi.org/10.1300/ J147v29n02_02

Frey, S., Neuenschwander, M. P., \& Nägele, C. (2016). Intention to participate in continuing education of young adults planning to have children concurrently Individual and situational aspects. Presented at the European Conference on Educational Resarch, Dublin, IE 23-26 August.

Grant, A. M., \& Ashford, S. J. (2008). The dynamics of proactivity at work. Research in Organizational Behavior, 28, 3-34.

Hackett, R. D., \& Lapierre, L. M. (2001). Understanding the links between work commitment constructs. Journal of Vocational Behavior, 58(3), 392-413. http://doi.org/10.1006/ jvbe. 2000.1776

Hansen, J.-I. C., \& Lee, W. V. (2007). Person-vocation fit. In S. G. Rogelberg (Ed.), Encyclopedia of Industrial and Organizational Psychology (pp. 621-623). Thousand Oaks, CA: SAGE Publications.

Heinz, W. R. (2000). Selbstsozialisation im Lebenslauf. Umrisse einer Theorie biografischen Handelns. In E. M. Hoerning (Hrsg.), Biografische Sozialisation (S. 165-186). Stuttgart, D: Lucius \& Lucius.

Holland, J. L. (1997). Making vocational choices. A theory of vocational personalities and work environments (3rd ed.). Odessa: Psychological Assessment Resources.

Jörin Fux, S. (2005). Persönlichkeit und Berufstätigkeit: Theorie und Instrumente von John Holland im deutschsprachigen Raum, unter Adaptation und Weiterentwicklung von Self-directed Search (SDS) und Position Classification Inventory (PCI). Göttingen, D: Cuvillier Verlag.

Jörin Fux, S., Stoll, F., Bergmann, C., Eder, F., \& Hell, B. (2012). EXPLORIX ${ }^{\oplus}$ - Das Werkzeug zur Berufswahl und Laufbahnplanung. Deutschsprachige Adaption und Weiterentwicklung des Self-Directed Search ${ }^{\varpi}$ (SDS) nach John L. Holland. Bern, CH: Verlag Hans Huber.

Judge, T. A. (2007). Perspectives on organizational fit. New York, NY: Psychology Press, Taylor \& Francis Group.

Kammeyer-Mueller, J. D., \& Wanberg, C. R. (2003). Unwrapping the organizational entry process: disentangling multiple antecedents and their pathways to adjustment. The Journal of Applied Psychology, 88(5), 779-794. http://doi.org/10.1037/0021-9010.88.5.779

Keller, A. C., \& Semmer, N. K. (2013). Changes in situational and dispositional factors as predictors of job satisfaction. Journal of Vocational Behavior, 83(1), 88-98. http://doi. org/10.1016/j.jvb.2013.03.004

Kristof, A. L. (1996). Person-organization fit: An integrative review of its conceptualizations, measurement, and implications. Personnel Psychology, 49(23), 1-49. http://doi. org/10.1007/978-3-531-90820-5

Kristof-Brown, A. L., Zimmerman, R. D., \& Johnson, E. C. (2005). Consequences of individuals' fit at work: A meta-analysis of person-job, person-organization, person-group, and person-supervisor fit. Personnel Psychology, 58, 281-342. 
Lee, K., Carswell, J. J., \& Allen, N. J. (2000). A meta-analytic review of occupational commitment: Relations with person- and work-related variables. Journal of Applied Psychology, 85(5), 799-811. http://doi.org/10.1037/0021-9010.85.5.799

Nägele, C., \& Neuenschwander, M. P. (2015). Passt der Beruf zu mir? Determinanten und Konsequenzen wahrgenommener Passung mit dem Lehrberuf beim Übergang in die Berufsbildung. In K. Häfeli, M. P. Neuenschwander, \& S. Schumann (Hrsg.), Berufliche Passagen im Lebenslauf (S.49-74). Wiesbaden, D: Springer Fachmedien. http://doi. org/10.1007/978-3-658-10094-0_3

Nägele, C., \& Neuenschwander, M. P. (2016). Apprentice-trainer relationship and work group integration in the first months of an apprenticeship. Empirical Research in Vocational Education and Training, 8(1), 4. http://doi.org/10.1186/s40461-016-0030-3

Neuenschwander, M. P., \& Düggeli, A. (2013). BEN Projektantrag: Berufliche Entscheidungen und Entwicklungsverläufe im Jugendalter und jungen Erwachsenenalter. Solothurn, $\mathrm{CH}$ : Fachhochschule Nordwestschweiz, Pädagogische Hochschule.

Neuenschwander, M. P., \& Frank, N. (2009). Familie-Schule-Beruf FASE B. Dokumentation der Schülerbefragung Erhebung 2008. Solothurn, CH: Fachhochschule Nordwestschweiz, Pädagogische Hochschule, Institut Forschung und Entwicklung, Zentrum Lernen und Sozialisation.

Neuenschwander, M. P., Düggeli, A., Nägele, C., \& Frey, S. (2015). Zweiter Zwischenbericht: Berufliche Entscheidungen und Entwicklungsverläufe im Jugendalter und jungen Erwachsenenalter (BEN). Solothurn, CH: Fachhochschule Nordwestschweiz, Pädagogische Hochschule, Institut Forschung und Entwicklung, Zentrum Lernen und Sozialisation.

Neuenschwander, M. P., Gerber, M., Frank, N., \& Rottermann, B. (2012). Schule und Beruf: Wege in die Erwerbstätigkeit. Wiesbaden, D: VS Verlag für Sozialwissenschaften.

Neuenschwander, M. P., Gerber, M., Frank, N., Singer, A., \& Bosshard, S. (2013a). (t1 - t5 L) Sozialisationsprozesse beim Übergang in den Lehrbetrieb (SoLe). Dokumentation der Lernendenbefragung. Zwischenerhebungen. Solothurn, $\mathrm{CH}$ : Pädagogische Hochschule der Fachhochschule Nordwestschweiz, Institut Forschung und Entwicklung, Zentrum Lernen und Sozialisation.

Neuenschwander, M. P., Hermann, M., Frank, N., \& Faschinger, S. (2013b). (W1 K4) Determinanten von Berufsbildungsentscheidungen beim Übergang in den Arbeitsmarkt (Ben). Dokumentation der Kohorte 4. Solothurn, CH: Fachhochschule Nordwestschweiz, Pädagogische Hochschule, Institut Forschung und Entwicklung, Zentrum Lernen und Sozialisation.

Neuenschwander, M. P., Nägele, C., \& Frey, S. (2016). Motivation to Participate in Formal Education during the School to Work Transition. Paper presented at the European Conference on Educational Research (ECER), 22.-26.8.2016, Dublin.

Nübling, M., Stößel, U., Hasselhorn, H. M., Michaelis, M., \& Hofmann, F. (2005). Methoden zur Erfassung psychischer Belastungen. Erprobung eines Messinstrumentes (COPSOQ). Dortmund, D, Berlin, D, Dresden, D: Bundesagentur für Arbeitsschutz und Arbeitsmedizin.

Parsons, F. (1909). Choosing a vocation. Boston, MA: Houghton Mifflin Company.

Savickas, M. L. (2012). Life design: A paradigm for career intervention in the 21 st century. Journal of Counseling \& Development, 90, 13-19.

Stalder, B. E., \& Nägele, C. (2015). Berufliche Identität, Commitment und Engagement. In M. Fischer, F. Rauner, \& Z. Zhao (Hrsg.), Kompetenzdiagnostik in der beruflichen Bildung - Methoden zum Erfassen und Entwickeln beruflicher Kompetenz (S. 259-273). Münster: LIT Verlag. 
Stalder, B. E., \& Schmid, E. (2016). Lehrvertragsauflösung und Ausbildungserfolg - kein Widerspruch. Bern, $\mathrm{CH}$ : hep verlag.

Super, D. E. (1980). A life-span, life-space approach to career development. Journal of Vocational Behavior, 16, 282-298.

Taris, T. W., Feij, J. A., \& Capel, S. (2006). Great expectations - and what comes of it: The effects of unmet expectations on work motivation and outcomes among newcomers. International Journal of Selection and Assessment, 14, 256-268.

Tims, M., Bakker, A. B., \& Derks, D. (2012). Development and validation of the job crafting scale. Journal of Vocational Behavior, 80(1), 173-186. http://doi.org/10.1016/j.jvb.2011.05.009

Trede, I. (2016). Laufbahnentscheidungen von Fachfrauen und Fachmännern Gesundheit nach Abschluss Ihrer Beruflichen Grundbildung. Bremen, D: Dissertation Universität Bremen.

Open Access Dieses Kapitel wird unter der Creative Commons Namensnennung 4.0 International Lizenz (http://creativecommons.org/licenses/by/4.0/deed.de) veröffentlicht, welche die Nutzung, Vervielfältigung, Bearbeitung, Verbreitung und Wiedergabe in jeglichem Medium und Format erlaubt, sofern Sie den/die ursprünglichen Autor(en) und die Quelle ordnungsgemäß nennen, einen Link zur Creative Commons Lizenz beifügen und angeben, ob Änderungen vorgenommen wurden.

Die in diesem Kapitel enthaltenen Bilder und sonstiges Drittmaterial unterliegen ebenfalls der genannten Creative Commons Lizenz, sofern sich aus der Abbildungslegende nichts anderes ergibt. Sofern das betreffende Material nicht unter der genannten Creative Commons Lizenz steht und die betreffende Handlung nicht nach gesetzlichen Vorschriften erlaubt ist, ist für die oben aufgeführten Weiterverwendungen des Materials die Einwilligung des jeweiligen Rechteinhabers einzuholen.

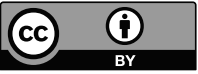

